\title{
SDN-POA Volume, Sexual Behavior, and Partner Preference of Male Rats Affected by Perinatal Treatment With ATD
}

\author{
E. J. HOUTSMULlER, ${ }^{* 1}$ T. BRAND, ${ }^{*}$ F. H. DE JONGE, $\dagger$ R. N. J. M. A. JOOSTEN, \\ N. E. VAN DE POLL $\ddagger$ AND A. K. SLOB*
}

\begin{abstract}
*Department of Endocrinology and Reproduction, Faculty of Medicine and Health Sciences, Erasmus University, P.O. Box 1738, 3000 DR Rotterdam. The Netherlands, †Department of Animal Husbandry, Section Ethology, Agricultural University Wageningen, P.O. Box 338, 6700 AH Wageningen, The Netherlands, and $\ddagger$ Department of Psychonomics, Faculty of Psychology, University of Amsterdam, Roetersstracct 15, 1018 WB Amsterdam
\end{abstract}

Received 10 June 1993

\begin{abstract}
HOUTSMULLER, E. J., T. BRAND, F. H. DE JONGE, R. N. J. M. A. JOOSTEN, N. E. VAN DE POLL AND A. K. SLOB. SDN$P O A$ volume, sexual behavior, and partner preference of male rats affected by perinatal treatment with ATD. PHYSIOL BEHAV 56(3) 535-541, 1994. - The present study investigated 1) the importance of the aromatization process during the perinatal period for the development of the sexually dimorphic nucleus in the preoptic area of the hypothalamus (SDN-POA) of male rats, and 2) the relationship between SDN-POA volume and parameters of masculinization in male rats that were treated perinatally with the aromatase-inhibitor ATD. Males were treated with ATD either prenatally or pre- and neonatally, or with the vehicle. Masculine sexual behavior and partner preference were investigated in adulthood. Thereafter, animals were sacrificed and SDN-POA volume was measured. The SDN-POA volume was reduced in both the prenatally and the pre- and neonatally treated group, with a larger reduction in the latter than in the former group. Combined pre- and neonatal ATD treatment resulted in reduced frequency of mounts, intromissions, and ejaculations, as well as a reduced preference for a female over a male. The SDN-POA size was significantly and positively correlated with frequency of masculine sexual behavior, as well as preference for a female over a male.
\end{abstract}

SDN-POA ATD Sexual behavior Perinatal Masculinization Male rats Sexual differentiation
Aromatization

RECENTLY it was found in our laboratory that perinatal treatment of male rats with the aromatase-inhibitor ATD (1,4,6-androstatriene3,17-dione), which blocks the aromatization of testosterone to estrogen, impairs masculine sexual behavior, enhances feminine sexual behavior, and decreases the preference for an estrous female over a sexually active male (6). The differentiation of masculine and feminine sexual behavior in various mammals has long been known to be directed by estrogens aromatized from androgens around birth $(4,20)$; in addition, partner preference behavior has now been suggested to be dependent on the same process [female ferrets (5), male rats (6), female rats (7)].

At the level of the central nervous system, the medial preoptic area (MPOA) of the hypothalamus has been strongly implicated as an important site for both gonadotropin release and the expression of masculine and feminine sexual behavior $(10,21,25,30,35)$. Within the MPOA, a sexually dimorphic nucleus (SDN-POA), which is severalfold larger in males than in females, was first described in rats $(18,19)$. A similar sex dimorphic nucleus has been subsequently identified in several other species [gerbil (11), guinea pig (22), ferret $(8,33)]$, including man $(13,32)$.
The sexual differentiation of the SDN-POA shows a close parallel with the sexual differentiation of behavior. First, the size of the SDN-POA of the adult rat is dependent on the presence of androgens during the perinatal period (24), as is behavioral sexual differentiation [see $(4,20)]$. Second, several findings suggest that the conversion of these androgens to estrogens perinatally is a prerequisite for the masculine development of the SDN-POA [(15), see also (16)]. The importance of the aromatization process during the perinatal period for behavioral sexual differentiation has also been well documented (4). Therefore, the first purpose of the present study was to investigate the importance of the aromatization process during the critical period for the development of the SDN-POA in male rats by determining the effect of perinatal treatment with the aromatase inhibitor ATD on SDN-POA volume in adulthood.

Because of its sex dimorphism, its location within the MPOA, and the parallels between its development and behavioral sexual differentiation, the specific involvement of the SDN-POA in the regulation of sexual behavior has been investigated in several

\footnotetext{
${ }^{1}$ Requests for reprints should be addressed to Els J. Houtsmuller, Department of Biological Sciences, University of Notre Dame, Notre Dame, IN 46556.
} 
studies. It has been found that the volume of the SDN-POA correlates significantly with sexual performance and testosterone levels in males (1). Moreover, lesions of this nucleus reportedly decrease masculine sexual behavior in several species, indicating the involvement of this nucleus in the expression of masculine sexual behavior [male rats (12), female rats (34), male gerbils (11), male ferrets (9) (although only small effects were found in this species)]. It should be noted, however, that negative findings have been reported as well (3).

Therefore, the second purpose of this study was to study SDN-POA volume in relationship to differences in partner preference and masculine sexual behavior, induced through perinatal ATD treatment. Subjects randomly drawn from a larger group of ATD-treated males described elsewhere (6) were reexamined following determination of SDN-POA volume.

\section{METHOD}

\section{Animals and Hormone Treatment}

Animals were housed two-four to a cage with food and water ad lib and kept on a reversed 14-10-h light-dark cycle.

Pregnant Wistar females received daily SC injection of ATD ( $5 \mathrm{mg}$ in $0.1 \mathrm{ml}$ propylene glycol) $(n=8)$ or propylene glycol $(0.1 \mathrm{ml})(n=4)$ from days $10-22$ of pregnancy (day of impregnation $=$ day 0 ). Within $9 \mathrm{~h}$ of birth, male pups from ATD mothers received a SC silastic implant (i.d. $1.5 \mathrm{~mm}$, o.d. $2.1 \mathrm{~mm}$, length $5 \mathrm{~mm})$ filled with ATD $(n=18)$, which was removed again after 21 days, or no implant $(n=12)$. Thus, three groups were formed: males that were treated pre- and neonatally with ATD (pn-ATD) $(n=18)$, males that were treated only prenatally with ATD (pre-ATD) $(n=12)$, and males for which mothers received injections of propylene glycol during pregnancy, and that served as control subjects (control) $(n=14)$. All animals were behaviorally tested in adulthood and the results have been described elsewhere (6). For the purpose of the present experiment, SDN-POA volume was measured in a subgroup of 15 animals, five drawn from each experimental group. This number proved sufficient to yield significant differences among groups. Selection of animals was random and did not take into account the results from the behavioral tests, because the primary goal of the present experiment was to determine the effect of perinatal ATD treatment on SDN-POA size. Had behavioral results been used as a selection criterion, it would not have been possible to distinguish between effects of experimental treatment on the one hand, and other, unknown variables that may have determined sexual behavior in these animals on the other hand.

Stimulus animals used in the behavioral tests were sexually active males and females brought into heat by $30 \mu \mathrm{g}$ EB (24-48 $\mathrm{h}$ before testing) and $2.5 \mathrm{mg} \mathrm{P}(3-4 \mathrm{~h}$ before testing). These hormones were dissolved in olive oil and injected SC.

\section{Procedure}

At 21 days of age, pups were weaned and housed two-four to a cage of same sex and treatment. They were left undisturbed until behavioral testing started, when the animals were 11 wk old.

Males were subjected to 13 weekly partner preference tests. In tests 1-7 no sexual interaction with stimulus animals was possible, because stimulus animals were separated from the experimental animals by wire mesh. In tests $8-13$ sexual interaction was possible. (See Behavioral Tests for details). Two weeks after the last preference test, the males were tested for masculine sexual behavior.
For purposes other than the present study, males were injected with 8-OH-DPAT prior to partner preference tests 6.10 , and 12 and the results of these tests are not included here. Prior to tests $4,5,9,11$, and 13 , animals were injected with a saline solution ( $1 \mathrm{ml}$ ). One week after the last partner preference test, males were tested for feminine sexual behavior, the results of which were presented elsewhere (6).

After all behavioral testing was completed, the animals were sacrificed, brains were weighed, and SDN-POA volumes were measured

\section{Behavioral Tests}

Partner preference. Partner preference tests were carried out in a test box with three compartments $(60 \times 30 \times 40 \mathrm{~cm}$ each) (31), in which a stimulus male was placed in one lateral compartment and a stimulus female in the other. Experimental animals could move freely from one compartment to the next through a small opening $(13 \times 12 \mathrm{~cm})$ in the partitions separating the compartments.

In the tests without interaction $(1-7)$, a wire mesh separated the experimental animals from the stimulus animals, allowing the animals to have nonphysical sensory contact with each other. In the tests with interaction (8-13), each stimulus animal was teth ered by a harness attached to the back wall of the compartment, limiting the action radius of the stimulus animals. In these tests physical interaction was possible between the experimental male and stimulus animals.

Prior to the test, stimulus and experimental animals were adapted to the test environment for $15 \mathrm{~min}$. During this adaptation time, the openings in the partitions were closed by sliding doors, so that no interaction between animals was possible. These doors were then removed, and for $15 \mathrm{~min}$ behaviors were observed through the transparent front. Time spent in each compartment was recorded for the experimental male, and when interaction was possible, mounts, intromissions, ejaculations, and lordosis were scored.

Masculine sexual behavior. The test for masculine sexual behavior was carried out in semicircular cages (radius $=36 \mathrm{~cm}$ ). Males were allowed a 5-min adaptation period before a stimulus female was introduced. For 15 min, mounts, intromissions, and ejaculations, as well as the latencies to these behaviors, were scored.

\section{Autopsy Procedures}

Upon completion of the behavioral tests, the animals were injected with pentobarbital (Nembutal, $0.5 \mathrm{ml} / \mathrm{rat}$, IP). All animals were then perfused intracardially with saline followed by $500 \mathrm{ml}$ fixative (4\% paraformaldehyde, $\mathrm{pH} 7.2$ ). The brains were removed and stored in fixative at $4^{\circ} \mathrm{C}$ for 1 day. Subsequently, the brains were dehydrated and embedded in paraffin. Serial 6- $\mu$ frontal sections were cut according to the coronal plane of the atlas of the brain of Paxinos and Watson (27), mounted upon chrome alum-coated slides and stained with thionin.

\section{Morphometry}

Area measurements of the cross-sectional SDN were performed bilaterally by means of a Calcomp 2000 digitizer connected to a VAX $11 / 780$ computer, using a Zeiss microscope with $\times 10$ and $\times 40$ (plan) objectives, respectively, and $\times 12.5$ plan oculars. The volume of the SDN was determined by integrating area measurements from the first to the last SDN cell-containing sections. To insure reliability, these measurements were taken twice under blind conditions, with a correlation of $0.70(p=$ 

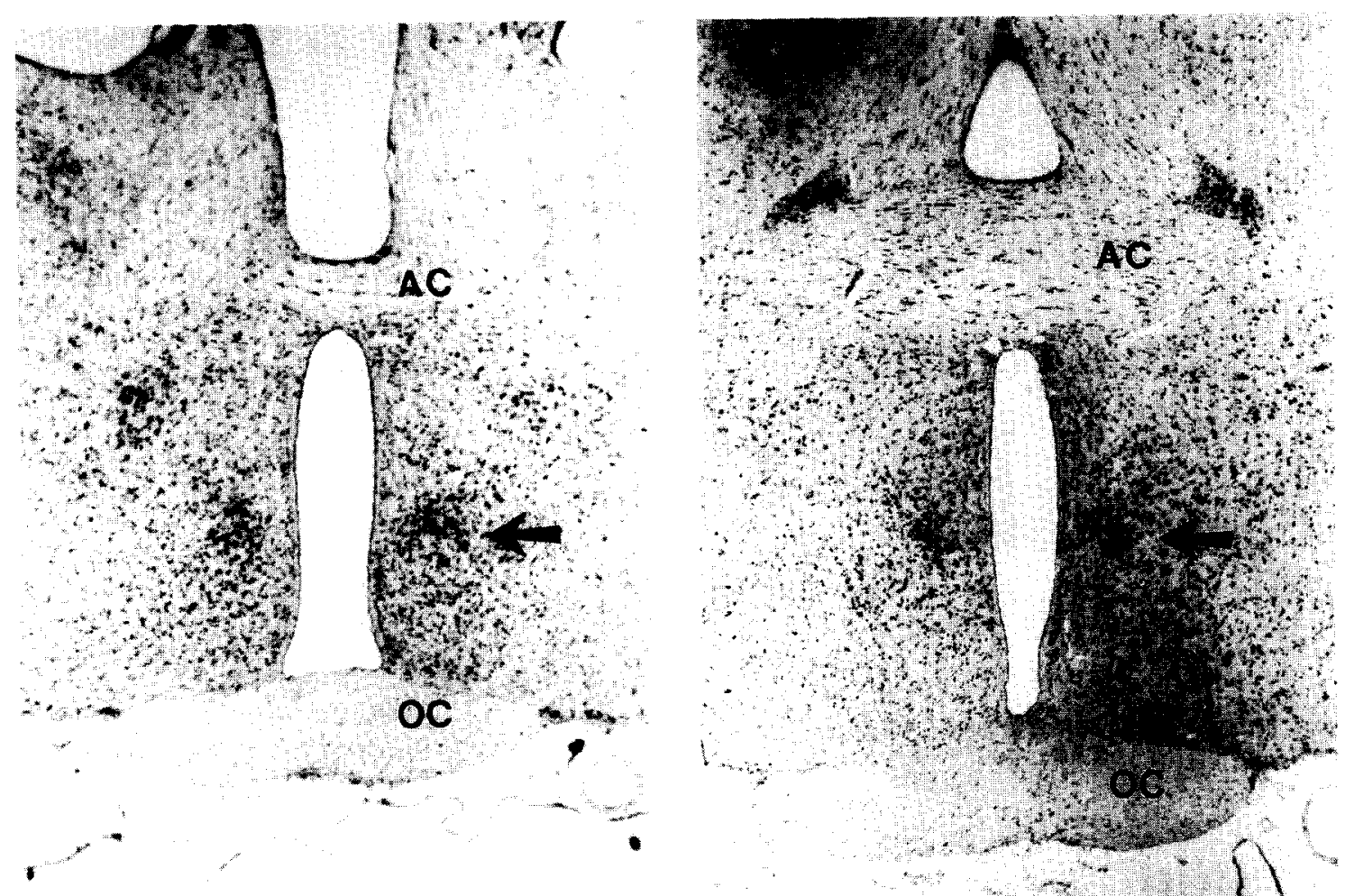

\section{A}
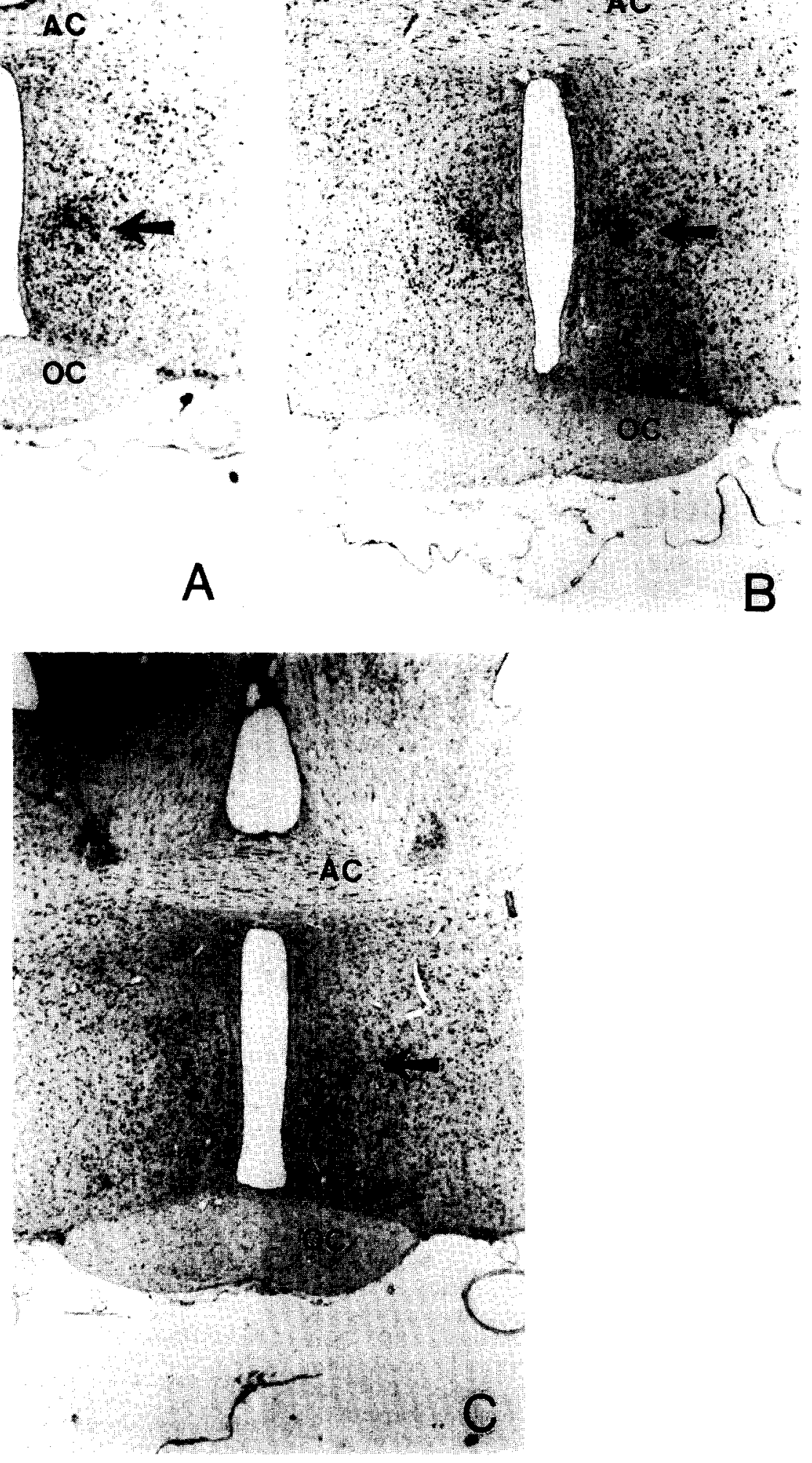

FIG. 1. Representative coronal sections of (A) a control male, (B) a male treated prenatally with ATD, and (C) a male treated pre- and neonatally with ATD. 


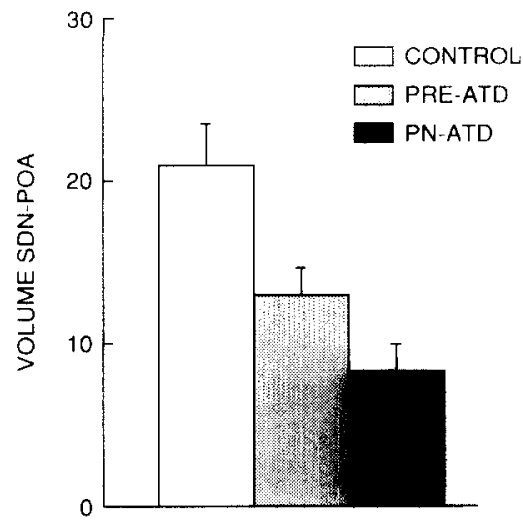

FIG. 2. Mean \pm SEM SDN-POA volume $\left(\times 10^{-3} \mathrm{~mm}^{3}\right)$ of males treated with ATD pre- and neonatally (pn-ATD), prenatally, (pre-ATD), and control males (control).

0.002 ) between the first and second measurement. The resulting mean of these two volumes was used in the data analysis.

\section{Data Analysis}

Data on SDN-POA volume and behavioral measures were subjected to a nonparametric analysis of variance (KruskallWallis), comparing the subgroups pn-ATD $(n=5)$, pre-ATD ( $n$ $=5$ ), and control $(n=5)$. When justified, individual group comparisons were made using the nonparametric Mann-Whitney test. Because there was overlap in SDN-POA volume between the groups, and because SDN-POA volume has been suggested to be a strong predictor of sexual activity in males (1), correlations between SDN-POA volume and several behavioral parameters were calculated in addition to the analyses of variance, which merely tested differences between groups. For these correlations, the three groups were combined as SDN-POA data comprised a normal distribution (Kolmogorov-Smirnov goodness of fit test, $p=0.96$, see Fig. 4). Correlations were calculated using the Pearson procedure (when data met the requirement of a normal distribution) or Spearman procedure (when this requirement was not met), and are reported only when significant. Differences in number of animals ejaculating were calculated using the Fisher Exact two-tailed probability test.

\section{RESULTS}

All animals appeared healthy throughout the experiment. Genitalia of males treated with ATD were normal in appearance at birth and continued to developed normally throughout the course of the experiment.

\section{SDN-POA Volumes}

Figure 1 shows a representative SDN-POA from each group. Mean volumes of the SDN-POA for the three groups are shown in Fig. 2. An overall significant difference between groups was found $(p=0.007)$. Subsequent analysis showed that SDN-POA volumes of the pn-ATD group were significantly smaller than those of both the pre-ATD and the control group $(p=0.047$ and 0.009 , respectively), and that SDN-POA volumes of the pre-ATD group were significantly smaller than those of the control group $(p=0.036)$. Mean brain weights (control: $1.9 \mathrm{~g}$, pre-ATD: 2.0 $\mathrm{g}$, pn-ATD: $1.9 \mathrm{~g}$ ) did not differ among groups (ANOVA, $p$ $>0.10$ ).
Behavioral Tests

Because there were statistically significant differences be tween the groups in SDN-POA volume, behavioral data of these subgroups were analyzed and correlated with SDN-POA volume.

Partner preference. To obtain a score for the preference for an estrous female, the time (in seconds) spent in the compartment of the stimulus female was divided by the total time spent in the compartments of the stimulus female and male. Figure 3 presents two mean preference scores, one for six tests in which physical interaction between experimental and stimulus animals was not possible, and one for four tests in which such interaction was allowed. There was no overall significant difference between the groups on mean preference score over tests without interaction (K-W, $p=0.174)$. Groups did differ significantly with respect to mean preference for a female on the tests with interaction $(\mathrm{K}-\mathrm{W}, p=0.009)$. Preference for a female on these tests was significantly lower for the pn-ATD group than both other groups $(p=0.009$ in both cases). No significant difference was found between the pre-ATD and the control group $(p=0.917)$. Combining over groups, SDN-POA volume correlated positively and significantly with preference for a female on both the tests without $(r=0.55, p=0.017)$, and with interaction $(r=0.66, p=$ $0.003)$.

Sexual behavior during partner preference tests with interaction. Latencies to the first mount or intromission did not differ across groups $(p=0.761,0.513,0.125$, and 0.745 on tests 8,9 . 11 , and 13 , respectively, means not shown).

Figure 4 shows the distribution of SDN-POA volumes and frequency of masculine sexual behavior during the partner preference tests across groups. Frequencies of mounts plus intromissions during the preference tests did not differ between groups on any of the tests $(p=0.128$; mean \pm SEM: pn-ATD $15.9 \pm$ 3.1 , pre-ATD $21.6 \pm 3.7$, control $23.3 \pm 1.2$ ). However, there was a significant correlation between frequency of mounts and intromissions, and SDN-POA volume $(r=0.67, p<0.01)$. In addition, mean number of ejaculations during these tests differed significantly across groups $(p=0.01)$, with animals from the pnATD group ejaculating less than both other groups $(p<0.04$ in both cases). The difference between the pre-ATD group and the

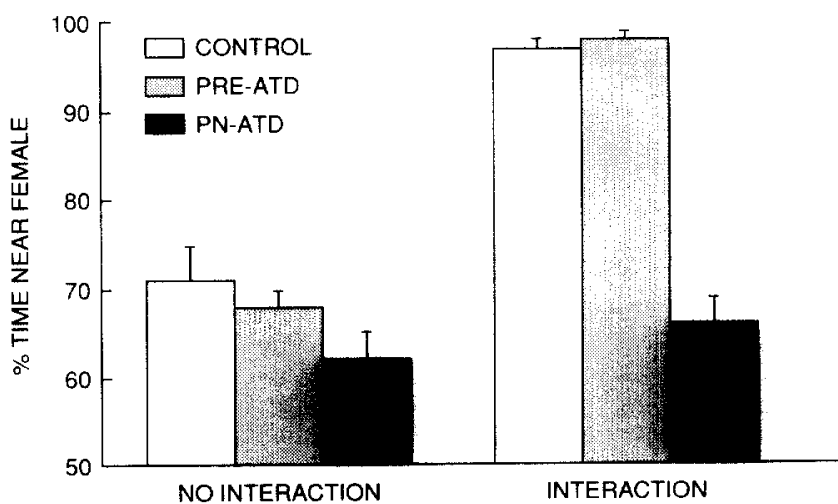

FIG. 3. Preference for an estrous female over a sexually active male, expressed as the mean \pm SEM amount of time spent near the female as a percentage of the total time spent near male and female. Bars represent a mean for six tests on which interaction was not possible as stimulus animals were separated from experimental animals by wire mesh (no interaction), and a mean for four tests in which stimulus animals were tethered and interaction between experimental animals and stimulus animals was possible (interaction). For explanation of treatments, see Fig. 2. 
MASCULINE SEXUAL BEHAVIOR DURING PREFERENCE TESTS
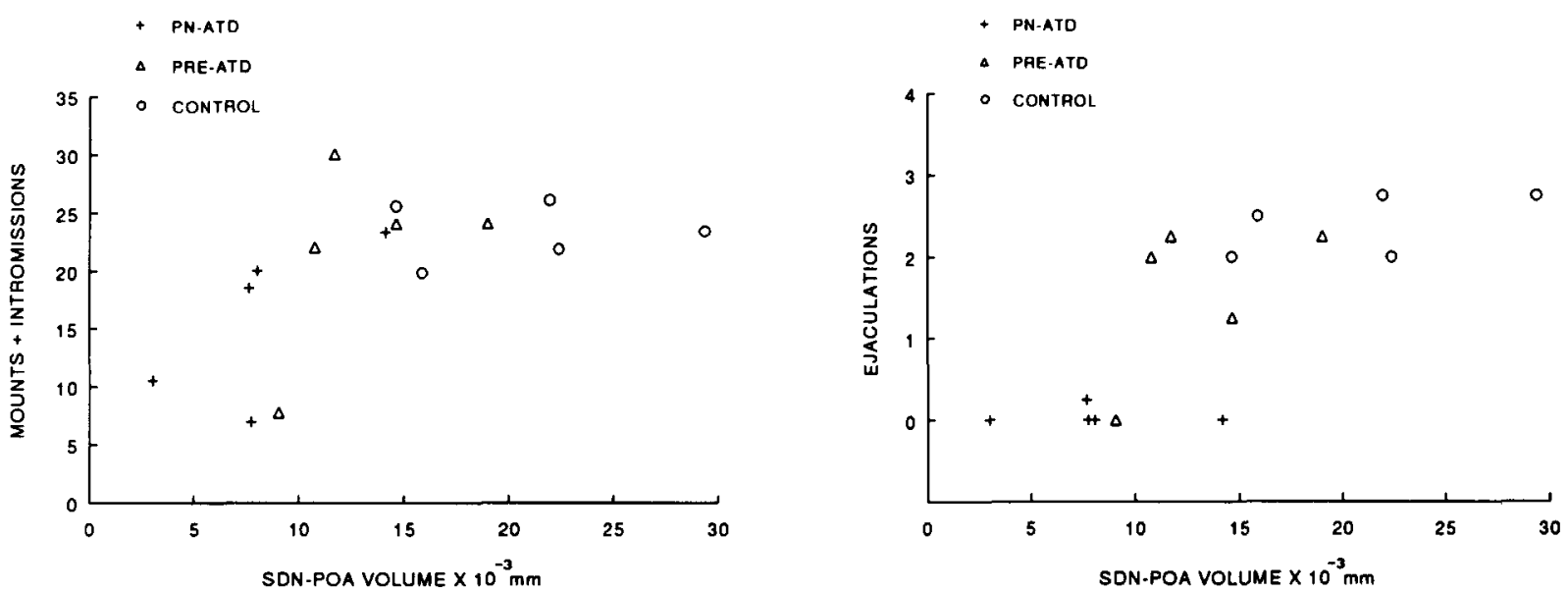

FIG. 4. Relationship between SDN-POA volume and mean number of mounts plus intromissions, and ejaculations for individual animals during four partner preference tests in which interaction with stimulus animals was possible. For explanation of treatments, see Fig. 2.

control group did not reach statistical significance $(p=0.11)$. The correlation between SDN-POA volume and mean number of ejaculations was positive and significant $(r=0.79, p<0.001)$.

Masculine sexual behavior during pair test. Mean number of mounts and intromissions during a test that paired the male with an estrous female for 15 min differed significantly across groups $(p=0.031)$ (Fig. 5). Mounts plus intromissions were significantly lower for the pn-ATD group than both the pre-ATD and the control group ( $p<0.04$ in both cases). Number of ejaculations was significantly different across groups as well $(p=$ 0.029 ). Animals from the pn-ATD group did not ejaculate and differed significantly from both other groups $(p<0.029$ in both cases). There was no difference between the pre-ATD group and the control group. SDN-POA volume and number of ejaculations during the pair test correlated significantly $(r=0.73, p=0.001)$.

\section{DISCUSSION}

The results of the present study show that in addition to behavioral sexual differentiation, SDN-POA volume of male Wistar rats is affected by perinatal treatment with the aromataseinhibitor ATD. The SDN-POA volume was significantly reduced by both pre- and perinatal (i.e., pre- + neonatal) treatment with ATD, with perinatal treatment being the most effective. The reduction in SDN-POA volume by combined pre- and neonatal ATD treatment was concomitant with reduced preference for an estrous female over a sexually active male and with reduced masculine sexual behavior. Furthermore, SDN-POA volume was positively and significantly associated with several parameters of masculine sexual behavior.

These results corroborate earlier studies, which suggest that the aromatization of androgens to estrogens perinatally is a prerequisite for the masculine development of the SDN-POA. For example, when administered perinatally, the nonsteroidal estrogen DES is as effective as testosterone in increasing SDN-POA size in female rats (14). Pre- and neonatal treatment with an estrogen antagonist reduced the size of the SDN-POA in males, whereas similar perinatal treatment with an antiandrogen did not have such an effect (15). In male ferrets, prenatal ATD treatment blocked the formation of a similar sex dimorphic nucleus (8). The conclusions from these reports are consistent with the results of the present study, which show that inhibition of aromatization of testosterone in males during the perinatal period significantly affects the development of the SDN-POA.
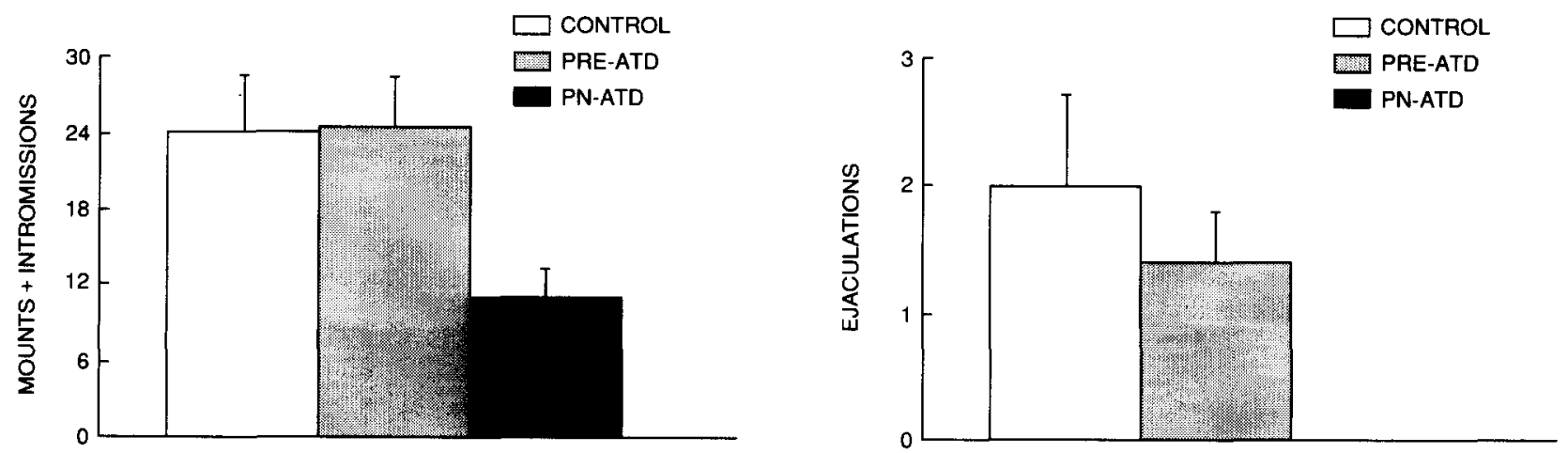

FIG. 5. Mean \pm SEM number of mounts, intromissions, and ejaculations during a test with a receptive female. For explanation of treatments, see Fig. 2. 
Combined pre- and neonatal ATD treatment was more effective in reducing SDN-POA volume than prenatal treatment alone. Several studies so far have indicated that the critical period during which the SDN-POA is sensitive to circulating gonadal hormones starts prenatally and extends into the neonatal period. That is, both prenatal and neonatal exposure to testosterone are effective in enlarging SDN-POA volume [e.g., prenatal $(2,23,28)$, neonatal $(24,29)]$. Moreover, neither neonatal androgen nor estrogen treatment is as effective as combined pre- and neonatal treatment in increasing SDN-POA volume (14). Our results are therefore consistent with the view that both pre- and neonatal exposures to estrogen are required for normal development of the SDNPOA in males. However, it cannot yet be ruled out that the greater effect in the combined pre- and neonatal treatment group might have resulted from the greater cumulative dose of ATD, as there was a longer period of treatment in this group.

Differences in SDN-POA volume were accompanied by major differences in behavior. That is, males that were pre- and neonatally treated with ATD, and thus had smaller SDN-POA volumes, showed a reduced preference for a female over a male on tests in which interaction with stimulus animals was possible. During these partner preference tests, they also showed reduced levels of masculine sexual behavior (no ejaculations). In addition, during a test in which males were paired with an estrous female, masculine sexual behavior was also impaired (fewer mounts and intromissions, and no ejaculations). These results reiterate the importance of the perinatal hormonal environment for partner preference, which has only recently been demonstrated in female ferrets (5), and male (6) and female rats (7), and further support the idea that perinatal exposure to estrogen aromatized from testosterone is a requirement for full behavioral masculinization of male rats [e.g., (4)]. The lack of differences between groups in partner preference scores where no interaction was possible is probably due to the low h's in the present experiment, as this difference was found in the population of rats from which our samples were drawn (6).

The concomitance of changes in SDN-POA volume and changes in behavior in the present study is significant because lesion and implantation studies have implicated the SDN-POA in the expression of masculine sexual behavior [rats $(13,24)$, gerbils (11)]. Although the effect of perinatal ATD treatment on behavior of male rats has been reported previously (6), a possible mechanism through which ATD might affect behavior, specifically through changes in SDN-POA volume, is suggested by our findings. The strong positive relationship between ATD-induced differences in SDN-POA volume and frequency of ejaculations corroborates an earlier report (1), which also indicated such a correlation. The present study expands this relationship to include mounts and intromissions. Taken together, these studies lend support to the idea that the SDN-POA may be involved in the expression of masculine sexual behavior. Interestingly, our results suggest that the subjects from our experiment in fact comprise two groups, one that ejaculates, the other that does not (see Fig. 4). Although not central to the present hypothesis, if two groups are established based upon behavioral criteria (ejaculators vs. nonejaculators), a significant difference in SDN-POA volume is found ( $p<0.02$, both for ejaculations during preference tests and during pair test). It appears that SDN-POA volume smaller than $10 \times 10^{-3} \mathrm{~mm}^{3}$ characterizes nonejaculators, whereas volumes larger than $16 \times 10^{-3} \mathrm{~mm}^{3}$ are typical of ejaculators.

Our data also provide evidence for a positive relationship between SDN-POA volume and preference for a female in tests with and without interaction. Measures of partner preference, when tested without the possibility of interaction with stimulus animals, are generally considered parameters of sexual motivation [e.g., (26)]. The SDN-POA thus far has been associated with consummatory aspects of sexual behavior (mounts, intromissions, and ejaculations), but not motivational aspects (17). In fact, partner preference of female rats was previously shown to remain unaffected by a lesion of the SDN-POA (34). Moreover, in the present study, latency to the first mount or intromission, a parameter that is typically considered a measure of motivation, did not correlate significantly with SDN-POA volume. However, the previous study, from which our sample of animals was drawn, did find a relationship between perinatal ATD treatment and time spent with a female when no interaction was possible (a measure of sexual motivation). Our finding that in males, larger SDNPOA volume is associated with a higher preference score for a female, even when no sexual interaction with the female is possible and thus consummatory aspects of sexual behavior are irrelevant, might serve to stimulate further research investigating a possible role for the SDN-POA in partner preference of males. Because it is clear that the relationship between SDN-POA volume and partner preference in our study lacks causal inference, further research, perhaps employing lesioning techniques that allow for establishing causal relationships, seems warranted. Finally, such studies should involve the SDN-POA as well as other brain structures sensitive to gonadal hormones.

Animals in the present study were left intact (i.e., were not castrated and it might be argued that the behavioral changes after perinatal ATD treatment could have resulted from differences in testicular output in adulthood. However, this is unlikely because perinatal ATD treatment previously has been shown to have no effect on adult androgen levels (6).

The results from the present experiment thus show a clear effect of perinatal ATD on SDN-POA volume in adulthood, and suggest a positive relationship between SDN-POA volume and consummatory aspects of masculine sexual behavior (mount, intromission, and ejaculation frequency) and to a lesser extent partner preference (time spent with a female when no physical interaction is possible). The correlations found between SDN-POA volume and several parameters of behavioral masculinization indicate that, in general, larger SDN-POA volumes were associated with increased behavioral masculinization. However, because of the limitations of a correlational design, further research is necessary to specify the relationship between SDN-POA volume, partner preference, and masculine sexual behavior. In addition, the relationship between more subtle variations in SDN-POA volume, as occurred within individual treatment groups, and behavioral parameters was not examined due to the low $n s$ in these groups. Future research needs to investigate possible behavioral differences associated with these more subtle differences in SDN-POA volume.

\section{ACKNOWLEDGEMEN'T}

The authors wish to thank Dr. D. L. Rowland for general comments on the manuscript, and Dr. D. F. Swaab for his consultation regarding measurements of the SDN-POA.

\section{REFERENCES}

1. Anderson, R. H.; Fleming, D. E.; Rhees, R. W.; Kinghorn, E. Relationships between sexual activity, plasma testosterone, and the vol- ume of the sexually dimorphic nucleus of the preoptic area in prenatally stressed and nonstressed rats. Brain Res. 370:1-10; 1986 
2. Anderson, R. H.; Rhees, R. W.; Fleming, D. E. Effects of prenatal stress on differentiation of the sexually dimorphic nucleus of the preoptic area (SDN-POA) of the rat brain. Brain Res. 332:113-118; 1985.

3. Arendash, G. W.; Gorski, R. A. Effects of discrete lesions of the sexually dimorphic nucleus of the preoptic area or other medial preoptic regions on the sexual behavior of male rats. Brain Res. Bull. 10:147-154; 1983

4. Baum, M. J. Differentiation of coital behavior in mammals: A comparative analysis. Neurosci. Biobehav. Rev. 3:265-284; 1979.

5. Baum, M. J.; Erskine, M. S.; Kornberg, E.; Weaver, C. E. Prenatal and neonatal testosterone exposure interact to affect the differentiation of sexual behavior and partner preference in female ferrets. Behav. Neurosci. 104:183-198; 1990.

6. Brand, T.; Kroonen, J.; Mos, J.; Slob, A. K. Adult partner preference and sexual behavior of male rats affected by perinatal endocrine manipulations. Horm. Behav. 25:323-341; 1991.

7. Brand, T.; Slob, A. K. On the organization of partner preference behavior in female Wistar rats. Physiol. Behav. 49:549-555; 1991.

8. Cherry, J. A.; Basham, M. E.; Weaver, C. E.; Krohmer, R. W.; Baum, M. J. Ontogeny of the sexually dimorphic male nucleus in the preoptic/anterior hypothalamus of ferrets and its manipulation by gonadal steroids. J. Neurobiol. 21:844-857; 1990.

9. Cherry, J. A.; Baum, M. J. Effects of lesions of a sexually dimorphic nucleus in the preoptic/anterior hypothalamic area on the expression of androgen- and estrogen-dependent sexual behaviors in male ferrets. Brain Res. 522:191-203; 1990.

10. Christensen, L. W.; Clemens, L. G. Intrahypothalamic implants of testosterone or estradiol and resumption of masculine sexual behavior in long-term castrated male rats. Endocrinology 95:984-990; 1979.

11. Commins, D.; Yahr, P. Lesions of the sexually dimorphic area disrupt mating and marking in male gerbils. Brain Res. Bull. 13:185$193 ; 1984$.

12. De Jonge, F. H.; Louwerse, A. L.; Ooms, M. P.; Evers, P.; Endert, E,; van de Poll, N. E. Lesions of the SDN-POA inhibit sexual behavior of male Wistar rats. Brain Res. Bull. 23:483-492; 1989.

13. De Jonge, F. H.; Swaab, D. F.; Ooms, M. P.; Endert, E.; van de Poll, N. E. Developmental and functional aspects of the human and rat sexually dimorphic nucleus of the preoptic area. In: Balthazart, J., ed. Hormones, brain and behaviour in vertebrates. 1. Sexual differentiation, neuroanatomical aspects, neurotransmitters and neuropeptides. Basel: Karger; 1990:121-136. (Comp. Physiol., vol. 8.)

14. Dohler, K. D.; Coquelin, A.; Davis, F.; Hines, M.; Shryne Gorski, R. A. Pre- and postnatal influence of testosterone propionate and diethylstilbestrol on differentiation of the sexually dimorphic nucleus of the preoptic area in male and female rats. Brain Res. 302:291-295; 1984.

15. Dohler, K. D.; Coquelin, A.; Davis, F.; et al. Pre- and postnatal influence of an estrogen antagonist and an androgen antagonist on differentiation of the sexually dimorphic nucleus of the preoptic area in male and female rats. Neuroendocrinology 42:443$448 ; 1986$.

16. Dohler, K. D.; Srivastava, S. S.; Shryne, J. E.; Jarzab, B.; Sipos, A.; Gorski, R. A. Differentiation of the sexually dimorphic nucleus in the preoptic area of the rat brain is inhibited by postnatal treatment with an estrogen antagonist. Neuroendocrinology 38:297-301; 1984.
17. Everitt, B. J. Sexual motivation: A neural and behavioral analysis of the mechanisms underlying appetitive and copulatory responses of male rats. Neurosci. Biobehav. Rev. 14:227-232; 1990.

18. Gorski, R. A.; Gordon, J. H.; Shryne, J. E.; Southam, A. M. Evidence for a morphological sex difference within the medial preoptic area of the rat brain. Brain Res. 148:333-346; 1978.

19. Gorski, R. A.; Harlan, R. E.; Jacobson, C. D.; Shryne, J. E.; Southam, A. M. Evidence for the existence of a sexually dimorphic nucleus in the preoptic area of the rat. J. Comp. Neurol. 193:529-539; 1980.

20. Goy, R. W.; McEwen, B. S. Sexual differentiation of the brain. Cambridge, MA: MIT Press; 1980.

21. Hart, B. L. Medial preoptic anterior hypothalamic area and the sociosexual behavior of male dogs: A comparative neuropsychological analysis. J. Comp. Physiol. Psychol. 86:328-349; 1974.

22. Hines, M.; Davis, F. C.; Coquelin, A.; Goy, R. W.; Gorski, R. A. Sexually dimorphic regions in the medial preoptic area and the bed nucleus of the stria terminalis of the guinea pig brain: A description and an investigation of their relationship to gonadal steroids in adulthood. J. Neurosci. 5:40-47; 1985.

23. Ito, S.; Murakami, S.; Yamanouchi, K.; Arai, Y. Prenatal androgen exposure, preoptic area and reproductive functions in the female rat. Brain Dev. 8:463-468; 1986

24. Jacobson, C. D.; Csernus, V. J.; Shryne, J. E.; Gorski, R. A. The influence of gonadectomy, androgen exposure, or a gonadal graft in the neonatal rat on the volume of the sexually dimorphic nucleus of the preoptic area. J. Neurosci. 1:1142-1147; 1981.

25. Malsbury, C. W. Facilitation of male rat copulatory behavior by electrical stimulation of the medial preoptic area. Physiol. Behav. $7: 797-805 ; 1971$.

26. Meyerson, B. J.; Lindstrom, L. Sexual motivation in the female rat. A methodological study applied to the investigation of the effect of estradiol benzoate. Acta Physiol. Scand. 88; Suppl. 389; 1973.

27. Paxinos, G.; Watson, C. The rat brain in stereotaxic coordinates. 2nd ed. Sydney, Australia: Academic Press; 1986.

28. Rhees, R. W.; Shryne, J. E.; Gorski, R. A. Onset of the hormonesensitive perinatal period for sexual differentiation of the sexually dimorphic nucleus of the preoptic area in female rats. J. Neurobiol. $21: 781-786 ; 1990$

29. Rhees, R. W.; Shryne, J. E.; Gorski, R. A. Termination of the hormone sensitive period for differentiation of the sexually dimorphic nucleus of the preoptic area in male and female rats. Dev. Brain Res. $52: 17-23 ; 1990$

30. Slimp, J. C.; Hart, B. L.; Goy, R. W. Heterosexual and sociosexual behavior of adult male rhesus monkeys with medial preoptic-anterior hypothalamic lesions. Brain Res. 142:105-122; 1978.

31. Slob, A. K.; de Klerk, L. W. L.; Brand, T. Homosexual and heterosexual partner preference in ovariectomized female rats: Effects of testosterone, estradiol and mating experience. Physiol. Behav. 41:571-576; 1987

32. Swaab, D. F.; Fliers, E. A sexually dimorphic nucleus in the human brain. Science 228:1112-1115; 1985.

33. Tobet, S. A.; Zahniser, D. J.; Baum, M. J. Sexual dimorphisms in the preoptic/anterior hypothalamic area of the ferret: Effects of adult exposure to sex steroids. Brain Res. 364:249-257; 1986.

34. Turkenburg, J. L.; Swaab, D. F.; Endert, E.; Louwerse, A. L.; van de Poll, N. E. Effects of lesions of the sexually dimorphic nucleus on sexual behavior of testosterone treated female Wistar rats. Brain Res. Bull. 21:215-225; 1988.

35. Van de Poll, N. E.; van Dis, H. Sexual motivation and medial preoptic self-stimulation in male rats. Psychon. Sci. 25:137-138; 1979. 